August 4-7, 2013, Portland, Oregon, USA

DETC2013-12497

\title{
PCB ORIGAMI: AMATERIAL-BASED DESIGN APPROACH TO COMPUTER-AIDED FOLDABLE ELECTRONIC DEVICES
}

\author{
Yoav Sterman \\ Mediated Matter Group \\ Media Lab \\ Massachusetts institute of \\ Technology \\ Cambridge, Massachusetts, \\ 02139 \\ sterman@mit.edu
}

\author{
Erik D. Demaine \\ Computer Science and Artificial \\ Intelligence Lab, \\ Massachusetts institute of \\ Technology \\ Cambridge, Massachusetts, \\ 02139 \\ edemaine@mit.edu
}

\author{
Neri Oxman \\ Mediated Matter Group \\ Media Lab \\ Massachusetts institute of \\ Technology \\ Cambridge, Massachusetts, \\ 02139 \\ neri@mit.edu
}

\begin{abstract}
Origami is traditionally implemented in paper of homogeneous material properties. This research explores the use of material with embedded electronics such as $P C B$ (Printed Circuit Boards) as the medium for origami folding in order to create an interactive folding experience and to generate foldable objects with added functionalities. PCBs are produced as $2 D$ shapes. By folding PCB arrays it is possible to create $3 D$ objects that contain electronic functions. Conductivity, output devices (such as Light Emitting Diodes) and microcontroller computation can create an interactive folding experience, for user guidance and verification of the folding. We call this approach and methodology PCB Origami. The work presented in this paper describes two unique interaction and fabrication techniques for creating and folding electronic materials. We demonstrate prototypes and present verification/evaluation strategies for guiding the user through the folding process.
\end{abstract}

\section{INTRODUCTION}

The practice of origami (paper folding) can be interpreted as a digital form-generation and fabrication process. A single sheet of paper can be folded into numerous shapes depending on folding sequence and crease positions [1]. Once the paper is folded it can be unfolded and folded again into a different shape. The physical (material) properties of the paper support its ability to fold and to unfold [2]. However, paper in itself is a passive material in the sense that it is not adaptive or responsive to external constraints, and the process of folding it is not interactive. The feedback one receives from folding paper is in most cases a visual one, comparing the physical sculpture to the drawing in the folding diagram.

In this work and research approach we propose using material with embedded electronics such as Printed Circuit Boards (PCB) as the medium for origami in order to create an interactive folding experience and to facilitate the design and fabrication of foldable products with integrated functionalities. PCBs are produced as two-dimensional (2D) shapes. By folding PCBs it is possible to create threedimensional (3D) objects that contain electronic functions such as light and sound performance. In addition, conductivity, LED (Light Emitting Diodes) output devices, 
and the computation of microcontrollers can by leveraged to create an interactive folding experience, for guiding the user in the folding procedure as compared to the planned sequence.

The work presented in this paper describes the interaction and fabrication techniques associated with the creation, assembly and folding sequence of electronic materials, as well as the strategies for guiding the user through the folding and the options to verify and evaluate the folding process itself.

\section{BACKGROUND}

Two main methods exist for guiding users in the process of folding a sheet of paper. They include the implementation of a folding diagram based on the Yoshizawa-Randlett system [3] and the use of a mountain valley diagram [4]. The Yoshizawa-Randlett folding diagram preserves the "digital" nature of paper-folding; according to this method a diagram is used to fold an origami sculpture. Once unfolded, the same sheet of paper can be used to fold another origami sculpture using a different diagram.

On the other hand, the Yoshizawa-Randlett folding diagram is not direct in the sense that it only provides a guiding external representation, and relies merely on the visual representation of the fold [3]. Printing a mountain valley diagram on the paper can be considered as a direct approach for guiding the user in the folding process, but it is not digital. Once the diagram is printed, it can only lead to the folding of a single shape.

In research, Interactive origami folding processes have been explored by representation systems that are external to the folding substrate (paper). The "origami desk" by Wu et al. [5] is an interactive installation that guides its users by projecting videos and images on the top of the working area. The system uses "hot spots" on the projected area as well as field sensing arrays, to collect inputs from the user and allows to him to fold in his own pace.

Another system, which uses a camera, is given by Kinoshita et al. [6], where the silhouette of the folded origami is analyzed for estimating the folding state. Both systems are based on external projection or imaging devices for receiving or transmitting information for the folding procedure.

An integrated approach for folding material and electronics was demonstrated in the self-actuated surface by Hawkes et al. [7]. The self-actuated surface automatically folds itself into multiple shapes depending on the inputs. Hawkes' research was the inspiration for exploring a material-based approach to origami folding, where cues associated with the folding procedure are embedded within the material substrate and promote the creation of a foldable object with integrated properties.

\section{APPROCH}

The approach taken in this work is a creation of an integrative system that uses both software and hardware. In this material-based approach [8], both the software and the hardware can be used as input and output in the folding process. The hardware refers to the folded PCB, which communicates with software installed on the computer. The folded PCB is embedded with LEDs and conductive connections. The LEDs are digital markers, indicating specific locations on the surface that are relevant for the current folding action. Conductive connections are being used to verify a connection was engaged, by checking the conductivity between two pads that are assumed to be connected. The conductive connection can be either momentary-two electrodes that touch each other-or temporary-creating a reversible bond that supports the structure of the folded piece.

Three types of possible temporary connection pads were developed: (1) magnetic, (2) mechanic or (3) thermal. (1) A magnetic connection uses metal magnets, such as neodymium magnets, for attachment and as conductive material, (2) Mechanical conductive connection is a metal spring or a press-fit that is soldered onto the PCB. An example of a mechanical conductive connection is the metal snap buttons common in fabrics. (3) The thermal conductive connection method utilizes a low temperature solder material that can be melted by heating up a nearby resistor. The resistor can be heated selectively depending on the folding sequence. The microcontroller mounted on the PCB senses that two pads are touching, then the solder material melts and the heating stops. After a few seconds the conductive bond is created.

\section{EXAMPLES}

Two examples demonstrating the concept of guided folding using PCB as the material are presented below. Each of these examples uses a different type of origami logic, a different material and different inputs and outputs methods.

The first example is based on the "Metamorphosis of the cube" by Demaine et al. [9], which demonstrates the idea of edge-to-edge gluing and folding of a flat polygon into convex polyhedra. In this example, a flexible PCB with LEDs and connection pads is laminated between two-dimensional machined sheets of acrylic plastic. The PCB and acrylic material are laminated, positioned and glued using pegs. After the gluing is complete, the Latin cross shaped 
polygon can be easily broken out of its frame (Figure 1). The result is a structure with rigid areas and hinges. The polygon can be folded into a cube or a different polyhedron depending on the sides being glued.
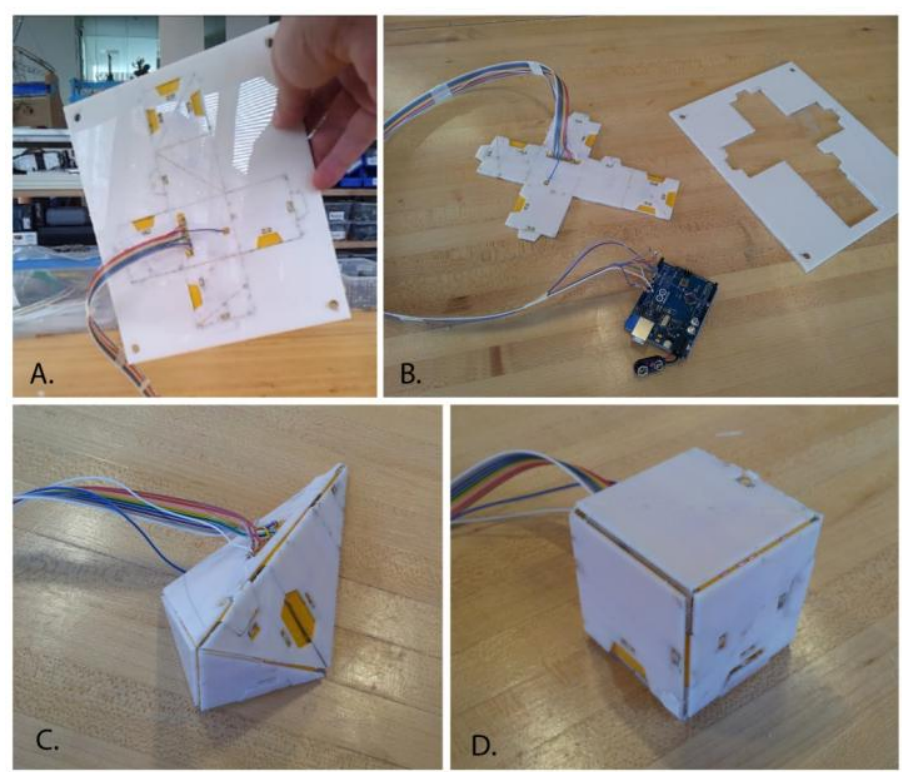

Figure 1. DEMONSTRATES THE PROCESS OF FABRICATING AND FOLDING THE LATIN CROSS POLYGON. A FLEXIBLE PCB IS LAMINATED BETWEEN TWO SHEETS OF MACHINED ACRYLIC PLASTIC (A). THE LATIN CROSS SHAPE IS CONNECTED TO THE FRAME WITH SMALL LINKS THAT CAN LATER BE BROKEN TO EXTRACT THE SHAPE (B). THE POLYGON CAN BE FOLDED INTO A PENTAHEDRON (C), OR A CUBE (D).

In this example, tabs are attached and slots are removed from some of the sides. For each shape that can be folded, a different set of tabs and slots must be connected in order to fold the shape (Figure 2). The folding process begins when the user selects the shape he wishes to fold with the software. Then, depending on the shape selected, the first set of LEDs blinks, marking the specific tab and the specific slot that needs to be connected. After the connection is made, the next set of LED blinks and the process continues until the folding is complete.

The second example uses software and paper covered with conductive pads in order to guide the user and verify the folding. The folded paper is a lamination of three layers of thin sheets of paper. The middle layer is being used as the circuit board (Figure 3). A circuit board made from copper stickers is attached to both sides of the paper.

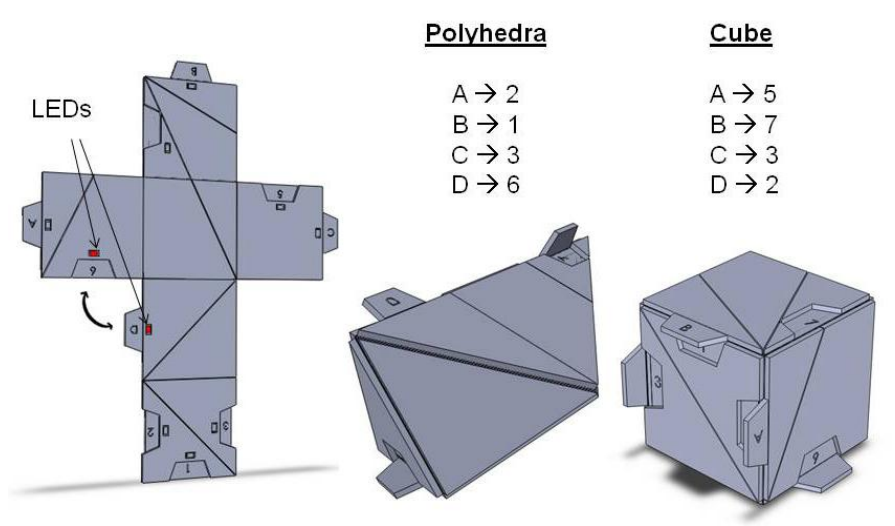

Figure 2. A SET OF TABS AND SLOTS HAVE BEEN PLACED ON SPECIFIC SIDES OF THE LATIN CROSS POLYGON. THE TABS ARE MARKED WITH LETTERS AND THE SLOTS ARE MARKED WITH NUMBERS. THE LISTS SHOW DIFFERENT PAIR ASSIGNMENTS, CREATING A PENTAHEDRON OR A CUBE. A LED IS PLACED NEXT TO EACH TAB OR SLOT.

The other layers are used for masking. Holes are cut in the outer layers using a laser cutter. After precision lamentation of the sheets, conductive circular stickers are glued on top of the holes and touch the circuit board on the middle layer.

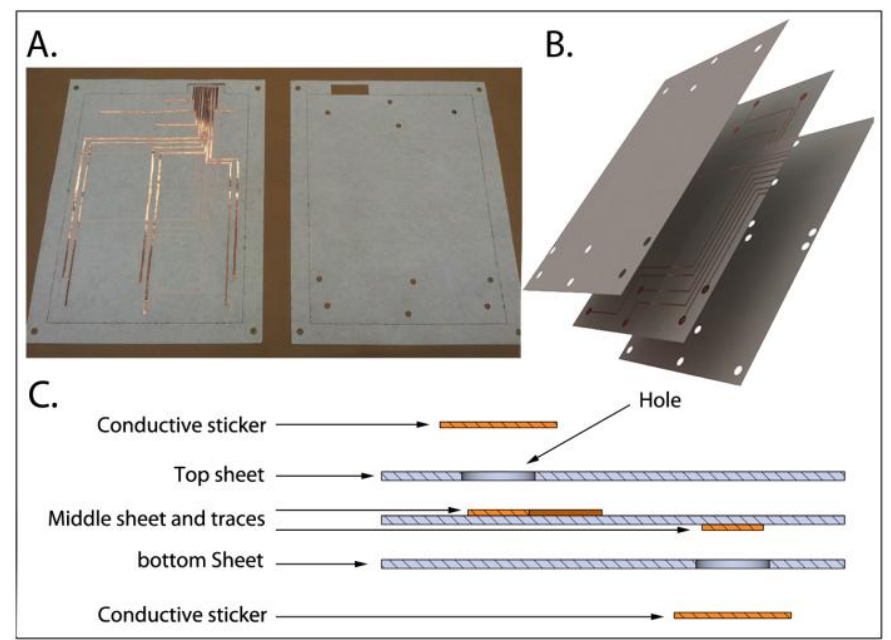

Figure 3. THREE LAYERS OF PAPER ARE LAMINATED USING PEGS. A CIRCUIT BOARD IS GLUED ONTO THE MIDDLE LAYER FROM BOTH SIDES (A) THE OUTER LAYERS ARE BEING USED FOR MASKING, WITH HOLES THAT REVEAL THE CONDUCTIVE PADS (B). STICKERS ARE GLUED ON THE OUTER SHEETS (CROSS SECTION IMAGE - C).

By connecting the paper to a microcontroller it is possible to know when two pads are touching each other (Figure 4). Each fold is perpendicular at the midpoint to the imaginary line that connects between the two pads. The user is guided through the folding process using the software. The software illustrates the folding with the circular pads on the paper as references. By checking 
conductivity between two pads it is possible to verify if the fold is correct, and move on to the next fold.

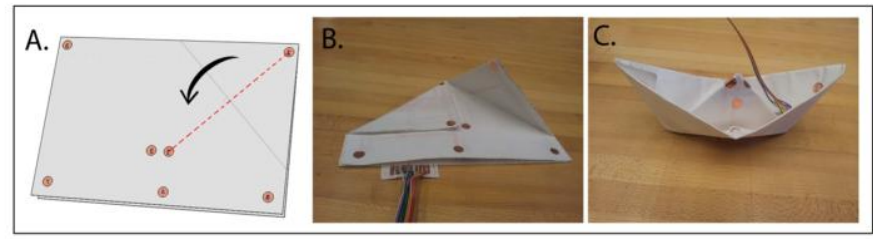

Figure 4. FOR EACH FOLD, A CREASE LINE IS PERPENDICULAR AT THE MIDPOINT TO A LINE BETWEEN TO DOTS (A). THE MICROCONTROLLER CAN SENSE WHEN TWO DOTS ARE TOUCHING (B), THEN SIGNAL THE SOFTWARE TO PROCEED TO THE NEXT FOLD. IN THIS EXAMPLE THE PAPER FOLDS INTO A BOAT, AFTER 12 STEPS (C).

\section{RESULTS}

In order to test the interaction and the quality of the guidance, a small user study of 10 participants was conducted. Participates were asked to fold the Latin cross shape into a polyhedron (figure 1-C), once guided by folding diagram and a second time using the LEDs. Result show that folding the shape using LEDs for guidance was three times faster than when a folding diagram was used (Table 1). On average, users spent 65 seconds folding the shape when guided by a diagram compared to 24 seconds when guided by LEDs. Participates reported a joyful experience, and communicated that they are more efficient and engaged in the process of folding. Folding using the diagram was described as "complicated" and "frustrating", while folding with guidance from LEDs was described as "super-obvious" and "easy". Even though, some of the participants liked the puzzle element of folding using a diagram and felt that the guidance from LEDs disturbs the solving process [10].

Table 1. USER STUDY RESULTS

\begin{tabular}{ccc}
\hline \#USER & $\begin{array}{c}\text { Time- guided by } \\
\text { diagram (seconds) }\end{array}$ & $\begin{array}{c}\text { Time- guided by } \\
\text { LEDs (seconds) }\end{array}$ \\
\hline 1 & 55 & 21 \\
\hline 2 & 45 & 20 \\
\hline 3 & 71 & 25 \\
\hline 4 & 88 & 38 \\
\hline 5 & 35 & 18 \\
\hline 6 & 65 & 20 \\
\hline 7 & 72 & 21 \\
\hline 8 & 86 & 28 \\
\hline 9 & 61 & 26 \\
\hline 10 & 72 & 23 \\
\hline
\end{tabular}

The tabs and slots method for "gluing" edges seemed to work well in the physical model of the Latin cross; the folded polyhedral shapes were solid and rigid. Beside the electronic contact property of the tabs and slot, its geometry also provided a mechanical connection that was helpful in the folding process. However, using tabs and slot limited the number of polyhedral shapes that are possible to fold. In "Metamorphosis of the cube", the Latin cross polygon is folded into five different polyhedra: a cube, a flat polygon and three convex polyhedra. In the physical model of the cross only a cube and a single other polyhedron can be folded. The reason is that folding other polyhedra, would require many more hinges, tabs, and slots."

\section{FUTURE WORK}

Future work will focus on other connection techniques that will allow greater flexibility in gluing options for polygons. Furthermore, we intend to design and fabricate additional and more complex polygons that can be folded into multiple polyhedral shapes based on edge-to-edge folding, and general gluing [11].

In the example of the conductive pads, the lamination of the three sheets of paper resulted in a material that was too thick and thus difficult to fold. Folds were too springy, consequently resulting in certain locations within the paper to "remember" the location of the fold. However, we were able to track the folding using the pads once the paper was connected to a microcontroller.

Future work will also improve the fabrication and lamination process of this material. Ink-jet printing on paper with conductive inks is a technology that is available today [12], and allows digital fabrication of flexible PCBs on flexible polyethylene terephthalate (PET) film. Also, as future work, we are interested in exploring a sheet of material with a matrix of dots in both sides that will report to a computer its folding state, depending on the pads that are touching each other. This sheet will enable users to "record" and save their folding sequences, review them later and also edit them.

\section{CONCLUSION}

The work presented in this paper introduces the concept of folding material with embedded electronics for making an interactive folding experience. As opposed to prior work in this field, this paper proposes a direct approach that does not rely on external imaging or projection devices, but rather on the interaction with the material itself. This kind of interaction is much more engaging and obvious for users. Indeed, in the user study conducted for this paper, users performed better when 
guided by the LEDs and reported a joyful folding experience.

\section{REFERENCES}

[1] Kanade, T., 1980, "A theory of origami world." Artificial Intelligence 13.3, pp. 279-311.

[2] Schenk, M., and Guest S.D., 2011, "Origami folding:A structural engineering approach." Origami 5: Fifth International Meeting of Origami Science, Mathematics, and Education. AK Peters Limited. pp. 291

[3] Lang, R. J., 1989, "Origami Diagramming Conventions: A His torical Perspective.", Robert J. Lang Origami. http://www.langorigami.com/diagramming/diagramming .php

[4] Kato, J., Shimanuki, H., and Watanabe, T,. 2008, "Automatically Making Origami Diagrams." Graphics Recognition. Recent Advances and New Opportunities. pp. 1-8..

[5] Ju, W., Bonanni, L., Fletcher, R., Hurwitz, R., Judd, T., Yoon, J., and Reynolds, M., 2001 "Origami Desk: Integrating Technological Innovation and Human centric Design". In Proceedings of DIS 2002. London: ACM Press, 2002, pp. 399-405

[6] Kinoshita, Y., and Watanabe, T., 2008, "Estimation of folding operation using silhouette of origami." IAENG International Journal of Computer Science 37(2).

[7] Hawkes, E., An, B., Benbernou, N. M., Tanaka, H., Kim, S., Demaine, E. D, Rus, D. \& Wood, R. J., 2010 ,
"Programmable matter by folding." PNAS 107(28) pp.12441-12445.

[8] Oxman. N., 2010, "Material-based design computation" $\mathrm{Ph} . \mathrm{D}$. thesis, Massachusetts Institute of Technolog Cambridge, Massachussests.

[9] Demaine, E.D., Demaine, M.L., Lubiw, A., O'Rourke, J., and Pashchenko, I. , 1999, "Metamorphosis of the cube." Proceedings of the 15th Annual Symposium on Computational Geometry. ACM. pp. 409-410.

[10] User study of folding a pentahedron out of Latin cross shape was conducted at the MIT Media Lab on December 4, 2012.

[11] Demaine, E. D., Demaine, M. L., Lubiw, A., and O'Rourke, J., 2002, "Enumerating foldings and unfoldings between polygons and polytopes." Graphs and Combinatorics 18(1) pp. 93-104.

[12] “Technology - Conductive Inkjet Technology “, http://www.conductiveinkjet.com, 2012

\section{ACKNOWLEDGMENTS}

This work was done as part of class 6.849: Geometric Folding Algorithms: Linkages, Origami, Polyhedra (Fall 2012) instructed by Prof. Erik Demaine at MIT, and in the context of Y. Sterman's master's thesis advised by Prof. Neri Oxman at the Mediated Matter group at the MIT Media Lab. 